\title{
A Survey of Multicasting in Optical Burst Switched Networks: Future Research Directions
}

\author{
Subhasis Datta \\ NSHM Knowledge Campus \\ Durgapur-713212, India
}

\author{
Avijan Dutta \\ National Institute of Technology \\ Durgapur-713209, India
}

\author{
Subhrabrata Choudhury \\ National Institute of Technology \\ Durgapur-713209, India
}

\begin{abstract}
A promising solution to increasing busty traffic over the Internet can be Optical Burst Switched (OBS) networks with scalable and efficient multicast support. The efficiency of multicasting in OBS networks depends on: the burstification process, the multicasting schemes, tree sharing strategies, construction of shared trees, multicast schemes for dynamic sessions and membership. In this article, the contributions of various researchers are studied thoroughly and compared to survey the various approaches and problems of multicasting in OBS networks and outline several future research directions in terms of applications in business, especially in Business-toBusiness (B2B) and Business-to-Consumer (B2C) Models, through optimal resource utilization of QoS aware multicasting in OBS networks.
\end{abstract}

\section{General Terms}

Optical Networks

\section{Keywords}

OBS, multicasting, tree sharing strategies, shared trees, multicast schemes, multicasting in OBS.

\section{INTRODUCTION}

Optical Burst Switching (OBS) technology is an effective technique to exploit the large bandwidth capacity of optic fibers to meet the exponentially increasing bandwidth requirement of the Internet. The inherent capability of extending statistical multiplexing gain makes it very suitable for providing high Quality of Service (QoS) in very high bandwidth network. Among which multicast services such as video-on-demand, video conferencing are becoming more prevalent. Multicast is a simultaneous transmission of information from one source to multiple destinations. Multicasting is a bandwidth-efficient technique because it prevents the source to send individual copies to each and every destination and also avoids flooding of the network by broadcasting.

\subsection{Optical Burst Switched Networks}

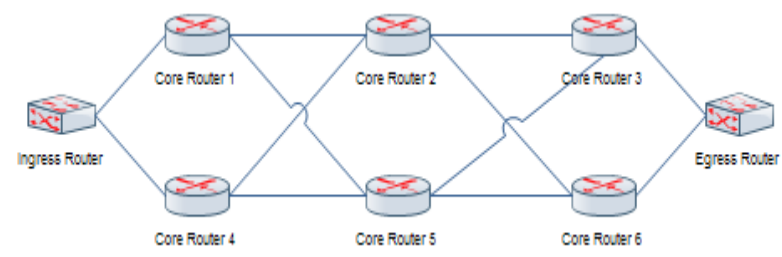

Fig. 1 Optical Burst Switched Network

All-Optical networks can be a promising solution for the explosive growth of the Internet traffic. Optical burst switching [1-5], among several other optical switching technologies, has gained a lot of attention recently because it combines the advantages of both circuit switching and packet switching. The features of Optical Packet Switching (OPS) and Optical Circuit Switching (OCS) have been utilized in OBS, to derive statistical multiplexing gain [1]. It is also advantageous for OBS, that no Optical/Electrical/Optical conversion is required at every intermediate router or switch. This is elegantly done by separating control functions and data transmission. In OBS networks [Fig 1.], the individual packets which are destined for a particular destination, is assembled into a burst. Researchers have proposed in [8-13], the process of burstification as time based with assembly time $\mathrm{Tb} \mathrm{m}$. It has been proposed in [19], that the min-burstlengthmax-assembly-period (MBMAP) algorithm, a burst is generated when a minimum burst length is reached or when the maximum assembly period is reached, whichever happens first. A Burst Header Packet (BHP) is created and sent ahead of the burst to reserve the optical path. The burst is then sent on a separate path (data channels) in the optical domain. The burst is then disassembled at the destination and forwarded to individual receivers. Recent implementation shows that the technology is not far away from aggressive commercial deployment and an All Optical Network may be based on OBS.

\subsection{Multicasting in Optical Burst Switched Networks}

With the increasing demand for multimedia over the internet, video-on-demand (VOD) or Audio-on-Demand (AOD) can be efficiently distributed to multiple clients through multicasting, by greatly improving the on-demand performance. Today's delivery systems are mostly unicast, where resources require linear scaling with the number of users and library sizes. Multicast multimedia systems are much more scalable and cheap to operate. A single stream is shared by a batch of common recipients/users, which makes this system more advantageous. As we are not far away to commercially implement an All Optical Network, with OBS technology, our focus of this paper will be a review on multicasting in OBS networks.

Several issues must be considered to multicast in OBS. First, a multicast tree or forest must be built for each multicast transmission. This problem has been addressed in [7] [8]. Similar ideas, but for wavelength routed networks were presented earlier in [6]. Second, a control packet needs to be sent before the transmission of each multicast burst. Third, the guard bands (GB) s waste the bandwidth, so we need multicast schemes to have a small overhead of GBs. We discuss several multicast schemes in optical burst switched networks, taking into consideration the overheads due to control packets and the GBs. For second and third issues it 
must be noted that, bandwidth consumption is directly proportional to the amount of guard bands. Channel utilization and processing overheads are directly proportional to the burst length and control packets generated respectively under same traffic conditions [11] [12].

In section II we discuss various multicast schemes proposed earlier and evaluate the performance of different schemes described earlier. Tree sharing multicasting is discussed in Section III. The various multicast schemes for dynamic membership is discussed in the section next, i.e. Section IV. In Section V, We conclude the paper in Section V with future research directions.

\section{MULTICASTING SCHEMES IN OPTICAL BURST SWITCHED NETWORKS}

The multicasting schemes of OBS are Separate Multicasting (S-MCAST), Multiple Unicasting (M-UCAST) and Tree Shared Multicasting (TS-MCAST) [11] [14]. It is assumed that each source maintains one burst assembly queue for each multicast session. The following schemes are for static multicast sessions.

\subsection{Separate Multicasting (S-MCAST)}

In S-MCAST, each multicast session (group) constructs its own source-specific multicast tree. The assembled bursts carrying multicast traffic for the group are delivered along with the multicast tree. Multicast traffic is transmitted independently of unicast traffic. With assembly time $T_{b}{ }^{m}$, the packets are assembled into a burst. After the assembly time is over, the burst is sent out along the multicast tree [12].

\subsection{Multiple Unicasting (M-UCAST)}

The next scheme is MUCAST. In this scheme, the multicast traffic of a group is delivered to all destinations through multiple unicast. During the assembly time of the burst, for each destination node in the multicast group, a copy of the multicast traffic will be assembled together with the unicast traffic. This forms a unicast burst for that node, if that traffic exists and then sent to that node. This reduces the overhead of GBs and control packets as the multicast traffic uses the control packets and GBs of unicast traffic. One of the drawbacks of this scheme is, it may result in low bandwidth efficiency because of the duplication of the multicast traffic. The network conditions dictate the overall performance of the scheme. But it is also possible that under certain network conditions, specifically considering the GB size, the MUCAST is better than SMCAST [11] [12].

\subsection{Tree Shared Multicasting (TS- MCAST)}

In TS-MCAST, there is a certain degree of membership overlap. Let $\mathrm{H}_{\mathrm{i}}$ be a set of all multicast sessions originating from edge router i. There may be also some special relationship between the multicast sessions. The set $\mathrm{H}_{\mathrm{i}}$ is split into number of subsets, based on some strategies, called Multicast Shared Class (MSC). Each subset or MSC may either construct a new shared tree (ST) or it may use one of the existing multicast trees, in other words, one of the multicast sessions of the subset. It depends on the algorithm used to create or select the ST. TS-MCAST uses one of the four tree sharing (when multiple multicast sessions use a single ST for the delivery of their multicast IP packets, we call it tree sharing) strategies namely, Equal Coverage, Super Coverage, Overlapping Coverage(OC) and Overlapping Coverage by Maximization (OCMAX). The burstification process allows the IP packets belonging to the multicast session in a MSC, to assemble together and form bursts.
Using TS-MCAST, the average burst length will be longer than without tree sharing. This will reduce the bandwidth waste due to GBs and less number of control packets will be generated. Here multicast traffic is transmitted independently of unicast traffic. To conclude, the multicast traffic of multiple sessions are mixed together to form a burst and that is then delivered by a shared multicast tree. The control packets and GBs are shared by multiple sessions and can achieve low overheads of control packets and GBs [11] [12]. It is clear from the conclusions of [11] [12] [18] that TSMCAST with the OC strategy shows the best performance, as the average path length increases or the effect of number of core routers. The performance of TS-MCAST (OC) is improved with the GB size. The performance of the two tree sharing strategies, EC and SC, improves drastically when the membership size is above 80 percent. TS-MCAST (OC) results in the best performance which consumes only a half of the bandwidth when compared to S-MCAST. The TSMCAST scheme achieves the best performance and shows improvement even with small number of multicast sessions. The TS-MCAST scheme performs best with average nodal degree compared to other schemes. But the performance of TSMCAST decreases as the amount of multicast traffic increases in the network. In [18] the Authors have clearly shown, that, it is reasonable to assign a longer burst assembly for multicast traffic as compared to unicast traffic time which increases the performance of the TS-MCAST. It has been also shown that TS-MCAST is particularly effective for traffic with low bandwidth when the GB size remains fixed and the same. TSMCAST can be applied to reduce the size of the multicast forwarding tables at each router in the network. TSMCAST can able to achieve a lower burst loss probability than SMCAST under similar traffic conditions [22].

So, it is seen that the proposed ST-MCAST [11] [12] [18] [22] performs better than other multicast schemes, than M-UCAST in all the cases and S-MCAST for criteria based on highest tree sharing gains. The ST-MCAST seems to be more viable as we are nearing to a commercial implementation of OBS.

\section{TREE SHARING MULTICASTING}

The tree sharing strategies describe the methods of decomposition of a set of multicast sessions into a number of MSCs, where each MSC uses a shared tree.

Let, $\mathrm{N}_{\mathrm{c}}$ be the set of all core routers. $\mathrm{N}_{\mathrm{e}}$ be the set of all edge routers. $\mathrm{N}_{\mathrm{l}}$ be the set of all links in the network. The Authors have modeled a multicast tree in the network using a triple $\mathrm{T}$ $=(\mathrm{C}, \mathrm{E}, \mathrm{L})$ where $\mathrm{C} \subseteq \mathrm{N}_{\mathrm{c}}$ is the set of core routers, $\mathrm{E} \subseteq \mathrm{N}_{\mathrm{e}}$ is the set of edge routers $\mathrm{L} \subseteq \mathrm{N}_{\mathrm{l}}$ is the set of links of the multicast trees. The network is modeled as a set of core routers, a set of edge routers, and a set of links connecting them. A multicast session is composed of an edge router that is the source, a set of some other edge routers that are the destinations, and a set of core routers and links that constitute the multicast tree[11][12]. When multiple multicast sessions use a single ST for the delivery of their multicast IP packets, we call it tree sharing.

\subsection{Tree Sharing Strategies}

In TS-MCAST, the set of multicast sessions originating from edge router is partitioned into a number of subsets, each of which is called a multicast sharing class (MSC) and uses a shared tree (ST). The IP packets from the multicast sessions in the same MSC are assembled together to form bursts. The major problem of TS-MCAST is then the partition strategy (or in other words, the tree sharing strategy). Our discussion starts with four tree sharing strategies mentioned above, Equal Coverage, Super Coverage, Overlapping Coverage (OC) and Overlapping Coverage by Maximization (OCMAX). These 
strategies are used to decide the subset of multicast sessions rooted at edge router, to become a MSC [11] [12]. The ideas are similar to as described by the authors in [15].

\subsubsection{Equal Coverage (EC)}

In EC, multicast sessions with the same membership, in other words, with the same set of member edge routers, are grouped into one MSC. So, s multicast sessions in MSCj have the same set of edge routers. Specifically, $\mathrm{E}_{\mathrm{i} 1}=\mathrm{E}_{\mathrm{i} 2}=\cdots=\mathrm{E}_{\mathrm{ij}}$, each multicast sessions in $\mathrm{MSC}_{\mathrm{j}}$ may have a different multicast tree.

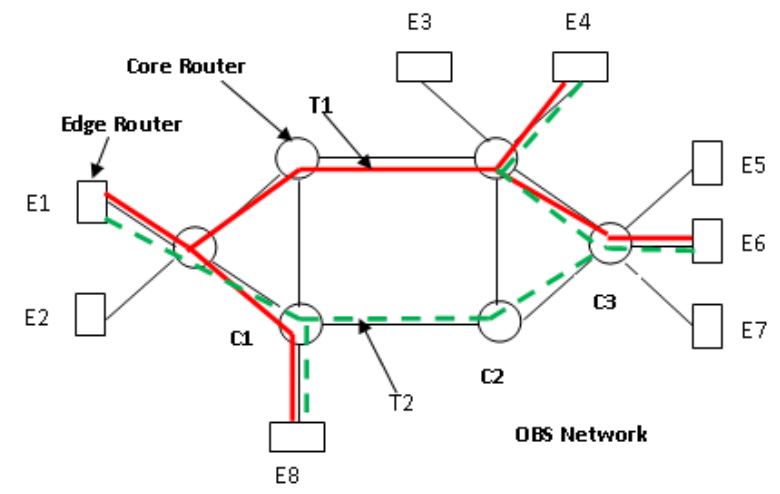

Fig. 2 Tree Sharing Strategy: Equal Coverage

The figure above (Fig. 2) shows an example of EC ( $s=2)$. Here multicast trees $\mathrm{T} 1$ (dashed red line) and T2 (dashed green line) have the same set of edge routers (E4, E6, E8) as their members and either T1 (solid red line) or T2 (dashed green line) is selected to be the new ST[11][12][14]. The same idea was given by the authors in [15] as Perfect Overlap (OP).

\subsubsection{Super Coverage (SC)}

If the set of member edge routers of a multicast session is a superset of that of another multicast session, these two multicast sessions are grouped into the same MSC having a tree sharing gain $\mathrm{j}$ above the threshold. In other words, SC is such a situation where EC is relaxed. That is, a number of multicast sessions to be grouped into one MSC does not need to have the same membership or the same number of edge routers.

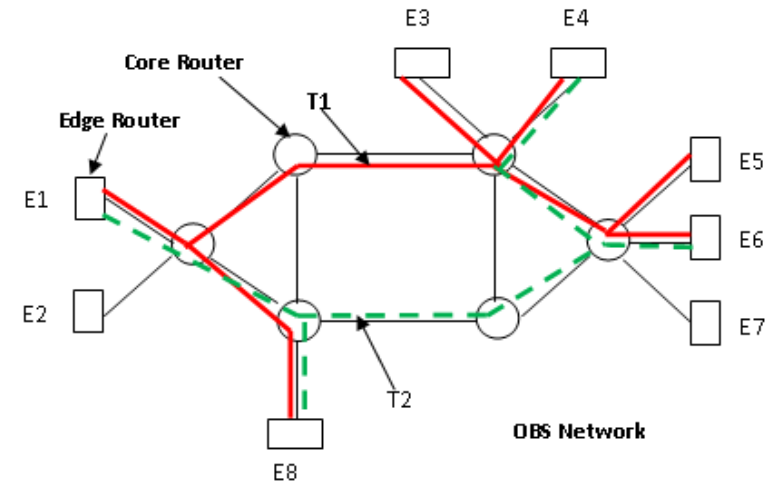

Fig. 3 Tree Sharing Strategy: Super Coverage

The multicast tree of the larger multicast session is selected as the new ST. Specifically, if two multicast sessions, T1 (solid red line) and T2 (dashed green line) have a special relation or in other words, T1 is a super tree for MSCj, such that ET2 $\subseteq$ $\mathrm{ET} 1$, then T1 and T2 are grouped into same $\mathrm{MSC}_{\mathrm{j}}$. The figure above (Fig. 3) shows the example of SC. The number of member edge routers can be one of the control parameters for
SC strategy [11] [12] [14]. The same proposition was also found in [15] in the name of Super Overlap (SO).

\subsubsection{Overlapping Coverage (OC)}

A number of multicast sessions having a sufficient degree of overlap in the edge routers, core routers, links, or tree sharing gain are grouped into the same MSC. There is a sufficient degree of overlap in edge routers $\mathrm{E}$, core routers $\mathrm{C}$, links $\mathrm{L}$ or tree sharing gain $\alpha$. Fig. 4 clearly shows the two multicast sessions T1 (denoted by dashed red line) and T2 (denoted by dashed green line) the concept of overlapping where both the trees are grouped into same MSC, if their (T1 and T2) combination has a traffic gain over a specified threshold based upon the criteria of edge router overlap, core router overlap, link overlap, and tree sharing gain.

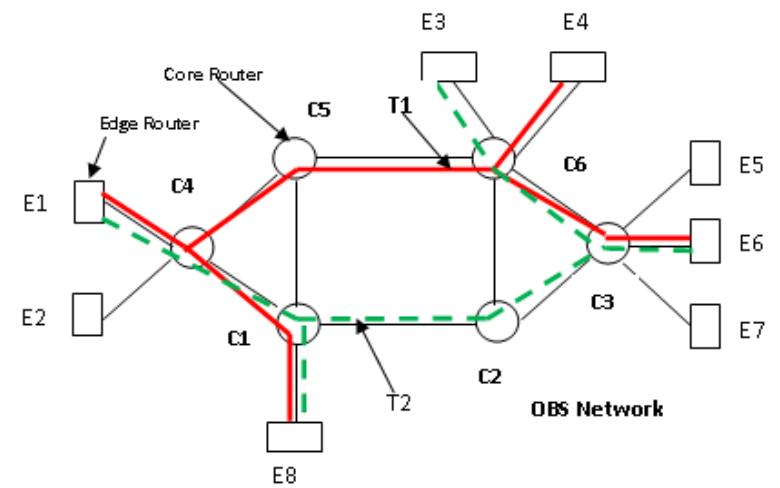

Fig. 4 Tree Sharing Strategy: Overlapping Coverage

The degree of overlap is the ratio of the number of nondistinct edge routers in the multicast trees to the number of distinct edge routers. More specifically, the degree of overlap is the difference between the sum of the number of edge routers in each multicast session and the number of all edge routers in the union of all multicast sessions, divided by the product of the number of edge routers in the union of all multicast sessions, and the number of multicast sessions minus 1[15].

The tree sharing gain can be defined as $\alpha_{j}$. The gain due to tree sharing $\alpha_{\mathrm{j}}$ is defined as the ratio of the average amount of multicast traffic carried per link without tree sharing to that with tree sharing. It reflects the amount of bandwidth that can be saved by tree sharing [11] [12] [14].

\subsection{Construction of Shared Trees}

After the multicast sessions Hi has been decomposed at the edge router $i$, into a number of MSCs using any of the above tree sharing strategies, the MSCs should construct a shared tree. This should be done by treating all the members in the subset of the multicast sessions as a new multicast group for the purpose of forming bursts and delivery of the bursts. Any of the existing trees can be used in EC and a super tree can be used in SC. But in OC and OC-MAX, the authors have proposed three algorithms to construct a shared tree.

\subsubsection{Greedy Algorithm}

This is a greedy algorithm where all the edge routers and core routers belonging to the existing multicast trees of a MSC are used to construct ST for MSC. In other words, this algorithm takes a union of all the existing multicast trees in the MSC. Though this approach is simple but it may output MSC with redundant links [11] [12].

\subsubsection{Breadth First Search Algorithm}

In this algorithm, BFS is applied to construct a ST, based on existing trees of a MSC. It starts at the edge source router, the root, and every adjacent node is examined to see if it is on the existing multicast tree but not yet in a ST. If so, both the link and the node are added to the ST. The node is also added to a 
queue for further consideration. This process is repeated until the queue is empty. Some redundant links of the Greedy Algorithm can be eliminated by this algorithm [11] [12].

\subsubsection{Member Initiated Algorithm}

In this member-initiated algorithm, an existing tree with the largest number of members is selected as the base of the new ST. All the other members join the ST by growing back toward the source along the links on the existing tree. This algorithm does not produce redundant links [11] [12] [14].

However, the authors in [14] have proposed the use of efficient Steiner heuristics such as [20] [21] to construct an efficient multicast tree for TS-MCAST.

\section{MULTICAST SCHEMES FOR DYNAMIC SESSIONS AND MEMBERSHIP}

Extensive study has been done by the authors in [12] for dynamic sessions and membership. The above schemes are also extended. They have focused mainly on the cases with dynamic birth and death of a session and dynamic join or leave of a member in an existing multicast session. Efficient heuristic algorithms are also proposed to manage dynamic sessions and membership under TS-MCAST schemes. The performance of three multicast schemes under limited bandwidth and dynamic sessions has been reported in [22], having their focus on burst blocking probability. The proposed heuristics and their performance are summarized.

\subsection{Dynamic Sessions}

For OC strategy, a session can be added dynamically to an existing multicast session or deleted dynamically from an existing multicast session at the edge router. For EC and SC, there is no checking of tree sharing gain. If a new session $n$ is created with predefined membership, then $n$ is evaluated with existing MSC. If the new session fits with the existing MSC, then $\mathrm{n}$ is added to the selected session. If a session expires, then the session and its related state information are deleted. However, after the deletion of the session the newly formed MSC is also evaluated. In [22], the details of the heuristics are reported.

\subsection{Dynamic Membership}

An edge router, commonly known as the member, can join or leave session at any time. First, the authors in [22] have differentiated the dynamic behavior into two cases, incremental change and decremental change. Then, to support dynamic join or leaving in a tree-shared multicasting, the authors have proposed two re-grooming approaches; time based approach and need based approach. In time based approach, the MSCs are evaluated at a regular interval to check for their tree sharing gain and in need based approach the MSCs are evaluated only if there is significant change in membership.

\subsection{Small Group Multicast with Deflection Routing}

The Authors have proposed in [23], a multicast scheme called OXCast, while studying the problem of small group multicasting in OBS. Multicast scheme was proposed based on deflection routing to reduce the data loss due to burst contention. An iterative network model was proposed by the Authors, based on reduced load approximation that could handle almost all the communication paradigms such as unicast and multicast traffic with or without deflection routing. Their performance analysis shows that there has been a reduction in burst loss probability but increases the burst delay.

\section{CONCLUSION AND FUTURE RESEARCH DIRECTIONS}

Most the research papers studied for this article, addresses the problem of Guard Bands and the number of Control Packets. More serious problems are still unaddressed and needs more attention with further research. We observe that each multicast session is to be mapped into a multicast spanning tree in the network meeting the individual QoS constraints of the session. If we have a number of such sessions setup sequentially in the network, then what should be the strategy so that at every point of time the unutilized bandwidth across the entire network is maximized. Alternatively, further research is needed to design a strategy that allows maximum number of QoS-aware multicast sessions in an OBS network with same level of resource requirement.

Elaborate research is needed to design QoS aware Multimedia Multicasting facilities in Optical Burst Switched networks that would result in optimal resource utilization for QoS aware multicasting in such network, having wide range of applications in business, especially in B2B and B2C Models. An analytical treatment of QoS-aware spanning trees and their impact on loss probability and traffic stability needs to be studied with fair accuracy. From the above study it is clear that the incremental bandwidth requirement for each session can be minimized.

We need to maximize the unutilized bandwidth not for a linear path from source to destination, but for a QoS aware multicast tree which is at least a spanning tree. Given a set of existing multicast sessions with specified QoS requirements (in terms of delay and loss) how should we configure/share/modify the multicast trees so that the unutilized bandwidth in the network is maximized.

Maximization of unutilized bandwidth will ultimately lead to dissemination of faster information and much more information in specified time duration. In a $\mathrm{B} 2 \mathrm{~B}$ or $\mathrm{B} 2 \mathrm{C}$ Model, this will result in providing more benefits to the end users at a much lesser cost (as cost per bit will go down for the service provider with maximum utilization of bandwidth). This indicates the value generating aspect of such technology utilization for the end users in a B2B or B2C model. Continuous effort of such maximization of even the slightest unutilized bandwidth will make this value generating process never-ending. Any business organization capable of doing that will always have a competitive advantage to sustain the potential competition in future.

\section{REFERENCES}

] C. Qiao and M. Yoo, "Optical burst switching (OBS)-a new paradigm for an optical Internet," Journal of High Speed Networks 8(1), pp. 69-84, 1999.

[2] M. Yoo, M. Jeong, and C. Qiao, "A high speed protocol for bursty traffic in optical networks, "in SPIEs AllOptical Communication Systems: Architecture, Control and Protocol Issues, 3230, pp. 79-90, 1997.

[3] J. Turner,"Terabit burst switching, "Journal of High Speed Networks 8(1), pp. 3-16, 1999.

[4] C. Qiao and M. Yoo,"Optical burst switching: A New Area in Optical Networking Research, "IEEE Network, pp. 16-23, May/June 2004.

[5] M. Yoo and C. Qiao, "A new optical burst switching (OBS) protocol for supporting quality of service," in SPIE Proc., All Optical Commun. Syst.: Architecture, Control Network Issues, vol. 3531, Nov. 1998, pp. 396405 . 
[6] X. Zhang, J. Wei, C. Qiao, "Constrained Multicast Routing in WDM Networks with Sparse Light Splitting,“ Proc. IEEE INFOCOMM '00, 2000, pp. 1781-90.

[7] Y. Xiong, M. Vandenhoute, H. Cankaya, "Control Architecture in Optical Burst-Switched WDM Networks," IEEE JOURNAL ON SELECTED AREAS IN COMMUNICATIONS, VOL. 18, NO. 10, OCTOBER 2000.

[8] X. Zhang, J. Wei, C. Qiao, "On Fundamental Issues in IP over WDM Multicast," Proc. Intl. Conf. Comp. Commun. and Networks (IC3N), 1999, pp. 84-90

[9] C. Qiao et al. "WDM Multicasting in IP over WDM Networks," Proc. Intl. Conf. Comp. Commun. and Networks (ICNP), 1999, pp. 89-96

[10] M. Jeong, C. Qiao, Y. Xiong, "Reliable WDM Multicast in Optical Burst-Switched Networks,"

[11] M. Jeong, Y. Xiong, H. Cankaya, M. Vandenhoute, and C. Qiao, "Efficient Multicast Schemes for Optical BurstSwitched WDM Networks," in IEEE ICC 2000, pp. 1289-1294, New Orleans, LA, June 2000.

[12] M. Jeong, C. Qiao, Y. Xiong, H. Cankaya, and M. Vandenhoute, "Bandwidth Efficient Multicast Schemes for Optical Burst-Switched WDM Networks," (submitted to journal)

[13] Myungsik Yoo, Chunming Qiao, and Sudhir Dixit, "QoS Performance of Optical Burst Switching in IP-OverWDM Networks," IEEE JOURNAL ON SELECTED AREAS IN COMMUNICATIONS, VOL. 18, NO. 10, OCTOBER 2000.

[14] M. Jeong, Y. Xiong, H. Cankaya, M. Vandenhoute, and C. Qiao, "Tree-Shared Multicast in Optical Burst-
Switched WDM Networks," Journal of Lightwave Technology, Vol. 21, No. 1, January 2003.

[15] M. Jeong, H. Cankaya and C. Qiao, "On New Multicasting Approach in Optical Burst-Switched Networks," IEEE Communications Magazine, 2002.

[16] H. Tode, H. Honda, K. Murakami, "Multicast Design Method Using Multiple Shared-Tree in OBS Networks"

[17] X. Huang, Q. She, T. Zhang, K. Lu, J. P. Jue, "Small Group Multicast with Deflection Routing in Optical Burst Switched Networks"

[18] M. Jeong, C. Qiao and M. Vandenhoute, "Distributed Shared Multicast Tree Construction Protocols for TreeShared Multicasting in OBS Networks"

[19] Cao, X., Li, J., Chen, Y., Qiao, C.: “Assembling TCP/IP packets in optical burst switched networks." In: Proceedings of IEEE GLOBECOM, pp. 8490 (2002)

[20] L. Kou, G. Markowsky, and L. Berman, "A fast algorithm for steiner trees," Acta Inform., vol. 15, pp $141145,1981$.

[21] M. Parsa, Q. Zhu, and J. J. Garcia-Luna-Aceves, "An iterative algorithm for delay-constrained minimum-cost multicasting," IEEE/ACM Trans. on Networking, vol. 6 , pp. 461474, Aug. 1998.

[22] M. Jeong, C. Qiao, Y. Xiong and M. Vandenhoute, "Evaluation of Multicast Schemes in Optical BurstSwitched Networks - the case with Dynamic Sessions," Proc. of SPIE, Terabit Optical Networking, vol. 2313 Nov. 2000.

[23] Xiaodong Huang, Qingya Shet, Tao Zhang, Kejie Lu, and Jason P. Juet, "Small Group Multicast with Deflection Routing in Optical Burst Switched Networks 